\title{
OPTIMIZED STEERABLE WAVELETS FOR TEXTURE ANALYSIS OF LUNG TISSUE IN 3-D CT: CLASSIFICATION OF USUAL INTERSTITIAL PNEUMONIA
}

\author{
Adrien Depeursinge ${ }^{\star \ddagger}$, Pedram Pad ${ }^{\ddagger}$, Anne S. Chin ${ }^{\dagger}$, Ann N. Leung ${ }^{\dagger}$, \\ Daniel L. Rubin ${ }^{\dagger}$, Henning Müller ${ }^{\star}$ and Michael Unser ${ }^{\ddagger}$ \\ ${ }^{\star}$ Institute of Information Systems, University of Applied Sciences Western Switzerland Sierre (HES-SO), \\ ${ }^{\ddagger}$ Biomedical Imaging Group (BIG), Ecole Polytechnique Fédérale de Lausanne (EPFL), Switzerland, \\ ${ }^{\dagger}$ Department of Radiology, Stanford University School of Medicine, CA, USA.
}

\begin{abstract}
Our aim is to optimize wavelet-based feature extraction for differentiating between the classical versus atypical pattern of usual interstitial pneumonia (UIP) in volumetric CT. Our proposal is to act on the bandwidth of steerable wavelets while maintaining their tight frame property. To that end, we designed a family of maximally localized wavelet pyramids in 3-D for a continuously adjustable radial bandwidth $[\Omega, \pi]$, $\Omega \in[\pi / 4, \pi / 2]$. The proposed wavelets are coupled with a rotation-covariant directional operator based on the Riesz transform, which provides characterizations of the organization image directions independently from their local orientations. The influence of the wavelet bandwidth on the classification performance was found to be large with area under the receiver operating characteristic curve (AUC) values in $[0.784,0.921]$. This demonstrated the importance of finding the minimum spatial support of the wavelet required to leverage the wealth of morphological tissue properties in the vicinity of the lung boundaries.
\end{abstract}

Index Terms - 3-D texture analysis, steerable wavelet pyramids, interstitial lung diseases.

\section{INTRODUCTION}

Texture analysis has been identified as the most promising approach to best leverage multi-dimensional morphological properties of biomedical tissue in radiological images [1]. It yields exhaustive, comprehensive and reproducible analysis of imaging features that are difficult to recognize with the naked eye in more than two dimensions. Local tissue structures are best modeled in terms of the multi-scale characterization of the organization of local image directions [2]. This requires the use of multi-scale image operators that are directional and can be locally oriented to normalize the distribution of image directions between all tissue samples mea-

This work was supported by the Swiss National Science Foundation (Grants 205320-141300/1, PZ00P2_154891) and the European Research Council (Grant ERC-2010-AdG 267439-FUN-SP). sured in a neighborhood $[3,4,5]$. Only few families of operators possess these properties. Among them are rotationinvariant local binary patterns and spherical harmonics [5], Gaussian Markov random fields [4], and steerable wavelet pyramids $[6,7,3]$.

The characterization of biomedical texture properties is tightly bound to the uncertainty relation for resolution in space, spatial frequency and orientation. The image operators need to be as localized as possible in space to capture local tissue properties and avoid the influence of surrounding objects (e.g., organ boundaries, vascular structures, see Fig. 1). On the other hand, the wealth of local texture information increases with the size of the texture operators used. It is therefore fundamental to optimize this trade-off in the context of a given medical application domain.

The optimization of this trade-off is of paramount importance for the automated differentiation of classic versus atypical usual interstitial pneumonia (UIP) in volumetric CT. The classic CT appearance of UIP is characterized with basal- and peripheral-predominant reticular abnormality and honeycombing [8]. An accurate characterization of the UIP pattern requires to first identify local alterations of the lung parenchyma, including reticulation, ground glass and honeycombing. The latter are characterized by local morphological distortions of the lung parenchyma. Second, the tissue types must be located in the lung anatomy (e.g., lung bases and periphery), since their presence only is not specific to the classic UIP pattern [9]. The image operators must therefore have a spatial support that is large enough to differentiate the tissue types and yet small enough to reduce the influence of the lung boundaries in both basal and peripheral regions (see Fig. 1). The current approach for identifying the classic UIP pattern in $\mathrm{CT}$ is to achieve multidisciplinary consensus of clinicians and radiologists with extensive experience in interstitial lung diseases. Additionally, a surgical biopsy is often required to confirm or reject the diagnosis. The procedure is invasive, costly and not without significant associated risks in patients with possible UIP. 


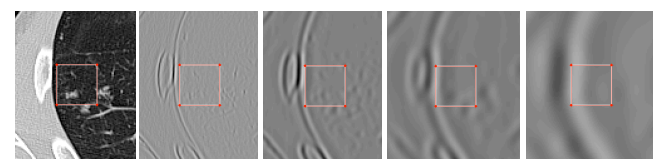

Fig. 1. Influence of nearby objects (e.g., lung boundaries) on local multi-scale texture properties in peripheral regions.

The contribution of this work is to extend the method proposed in [10] to 3-D to design a family of wavelet of maximally localized wavelet pyramids over the radial bandwidth $[\Omega, \pi]$ for $\Omega \in[\pi / 4, \pi / 2]$. The tuning of the radial bandwidth of the steerable wavelet pyramid is used to optimize the trade-off between the spatial support of the wavelets and the wealth of the texture information modeled. The optimization of the wavelet bandwidth is coupled with the alignment of Riesz operators to maximize their local energy. This allows characterizing the multi-scale organization of image directions independently from their local orientation (referred to as "rotation-covariance" $[12,3])$. The medical objective of this work is to provide reliable and reproducible computerized assistance for the identification of the UIP pattern in less specialized practice centers without access to experts experienced in interstitial lung disease. Nevertheless, reduction of invasive biopsies is desired when possible to reduce costs and avoid unnecessary morbidity in UIP patients.

\section{MATERIAL AND METHODS}

\subsection{Patients}

33 patients from Stanford Hospital and Clinics with biopsyproven UIP were retrospectively reviewed in consensus by two thoracic radiologists with more than 15 years of experience. Based on the American Thoracic Society evidencebased guidelines for CT diagnosis [8], 15 cases were identified as having a classic UIP pattern and 18 were atypical. A volumetric CT scan was available for each patient, which was acquired within the year of the biopsy proven diagnosis of UIP. All volumes were resampled to have isotropic voxels of $0.59 \times 0.59 \times 0.59 \mathrm{~mm} 3$ using cubic spline interpolation.

\subsection{Rotation-covariant texture analysis}

\subsubsection{3-D Riesz-wavelet frames}

3-D multi-scale Riesz filterbanks are used to characterize the local volumetric texture properties of the lung parenchyma in CT. The Riesz transform $\mathcal{R}$ of finite-energy signals $f(\boldsymbol{x}) \in$ $\mathbb{R}^{3}$ is defined in the Fourier domain as:

$$
\widehat{\mathcal{R} f}(\boldsymbol{\omega})=\frac{-j \boldsymbol{\omega}}{\|\boldsymbol{\omega}\|} \hat{f}(\boldsymbol{\omega}),
$$

where $\hat{f}(\boldsymbol{\omega})=\int_{\mathbb{R}^{3}} f(\boldsymbol{x}) e^{-j\langle\boldsymbol{w}, \boldsymbol{x}\rangle} \mathrm{d} \boldsymbol{x}$ is the 3-D Fourier transform of $f$ with $\omega \in \mathbb{R}^{3}$. It yields three Riesz components $\mathcal{R}_{i}$ that can be viewed altogether as a localized gradient operator when coupled with an isotropic wavelet pyramid [7]. This operator commutes with translation, scaling and rotation and is therefore an excellent candidate to derive localized multiscale directional measures of 3-D texture properties [1].

\subsubsection{Steerability and rotation-covariance}

The Riesz filterbank obtained from (1) is steerable, meaning that the magnitude of each Riesz operator $\mathcal{R}_{i}$ oriented in any direction can be obtained from a linear combinations of the initial operators. Specifically, $\mathcal{R}\{f\}(\boldsymbol{U} \boldsymbol{x})=\boldsymbol{U} \mathcal{R}\{f\}(\boldsymbol{x})$, where $\boldsymbol{U}$ is a unitary rotation matrix [11]. The steerability is used to normalize orientations at each voxel $\boldsymbol{x}_{0}$ by rotating the Riesz operator with $\boldsymbol{U}_{g}$ in order to maximize its local energy. $\boldsymbol{U}_{g}$ is derived from the sorted collection of eigenvectors of the tensor matrix $\boldsymbol{J}\left(\boldsymbol{x}_{0}\right)$ defined as:

$\boldsymbol{J}\left(\boldsymbol{x}_{0}\right)=\left(\begin{array}{ccc}\mathcal{R}_{1}^{2}\{g * f\}\left(\boldsymbol{x}_{0}\right) & \mathcal{R}_{1} \mathcal{R}_{2}\{g * f\}\left(\boldsymbol{x}_{0}\right) & \mathcal{R}_{1} \mathcal{R}_{3}\{g * f\}\left(\boldsymbol{x}_{0}\right) \\ \mathcal{R}_{1} \mathcal{R}_{2}\{g * f\}\left(\boldsymbol{x}_{0}\right) & \mathcal{R}_{2}^{2}\{g * f\}\left(\boldsymbol{x}_{0}\right) & \mathcal{R}_{2} \mathcal{R}_{3}\{g * f\}\left(\boldsymbol{x}_{0}\right) \\ \mathcal{R}_{1} \mathcal{R}_{3}\{g * f\}\left(\boldsymbol{x}_{0}\right) & \mathcal{R}_{2} \mathcal{R}_{3}\{g * f\}\left(\boldsymbol{x}_{0}\right) & \mathcal{R}_{3}^{2}\{g * f\}\left(\boldsymbol{x}_{0}\right)\end{array}\right)$,

where $g(\boldsymbol{x})$ is a 3-D Gaussian window. For each location $\boldsymbol{x}_{0}$, the resulting rotation matrix $\boldsymbol{U}_{g}$ maximizes the energy of $\mathcal{R}_{1}\{g * f\}\left(\boldsymbol{x}_{0}\right)$ as well as the residual energies of $\mathcal{R}_{2}\{g *$ $f\}\left(\boldsymbol{x}_{0}\right)$, followed by $\mathcal{R}_{3}\{g * f\}\left(\boldsymbol{x}_{0}\right)$.

This property is referred to as "rotation-covariance" (represented by $\boldsymbol{\mathcal { R }}^{\mathrm{RC}}\{f\}(\boldsymbol{x})$ ), where the organization of image directions is characterized independently from its local orientation $[12,3]$. The latter differs from the monogenic signal [11] since the entire pyramid is using the same rotation matrix $\boldsymbol{U}_{g}$ derived from the highest image resolution regularized by $g(\boldsymbol{x})$. It also differs from rotation-invariant operators as $\mathcal{R}^{\mathrm{RC}}\{f\}(\boldsymbol{x})$ is directional.

\subsection{Isotropic wavelet pyramids with optimal bandwidth}

In order to measure local directional properties of the lung tissue at $M$ consecutive physical scales, the Riesz transform must be coupled with a primary wavelet pyramid. 3-D Rieszwavelet frames are built by applying the Riesz transform to each scale of the wavelet pyramid given by a wavelet function $\psi(\boldsymbol{x}): \boldsymbol{\mathcal { R }}\left\{\psi_{m} * f\right\}(\boldsymbol{x})$ for $m=1, \ldots, M$. The design of the wavelet pyramid must be carried out to fulfill particular conditions, which cannot be satisfied altogether by conventional orthogonal and separable wavelet transforms [13]. The main property is that it must be isotropic to preserve the directional decomposition of the Riesz transform. Some other properties like band limitedness are also necessary $[14,15,13]$.

Under these constraints, we designed the wavelet that is maximally localized to minimize the influence of surrounding objects (i.e., lung boundaries, bronchovascular structures) on the local texture properties. Therefore, we used the method proposed in [10] for designing maximally localized isotropic wavelets but for 3-D. Moreover, since experiments showed 


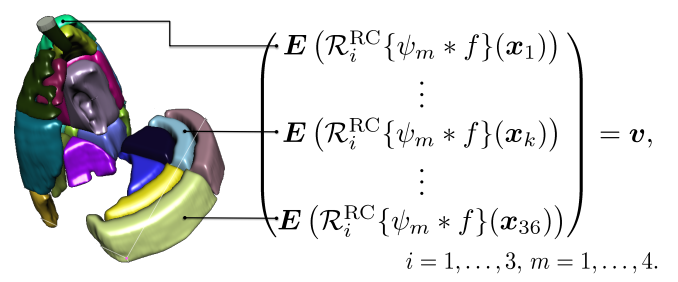

Fig. 2. Construction of the feature vector $\boldsymbol{v}$ from regional averaging of the energies $\boldsymbol{E}\left(\mathcal{R}_{i}^{\mathrm{RC}}\left\{\psi_{m} * f\right\}(\boldsymbol{x})\right)$ [9].

that the Papadakis wavelet [13] with a radial bandwidth of $[3 \pi / 10, \pi]$ outperformed other proposed wavelets like Simoncelli [6], Meyer [16] that have radial bandwidth of $[\pi / 4, \pi]$, we designed a family of maximally localized wavelets over the radial bandwidth $[\Omega, \pi]$ for $\Omega \in[\pi / 4, \pi / 2]$ to simultaneously find the optimal bandwidth. The outcome of our design is the following Fourier-domain specification of our new wavelets:

$$
\widehat{\psi}_{\Omega}(\boldsymbol{\omega})= \begin{cases}0 & \|\boldsymbol{\omega}\| \leq \Omega \text { or } \pi<\|\boldsymbol{\omega}\|, \\ \sqrt{\frac{\log \frac{\|\boldsymbol{\omega}\|}{\Omega}}{\log \frac{\pi}{2 \Omega}}} & \Omega<\|\boldsymbol{\omega}\| \leq \frac{\pi}{2} \\ 1 & \frac{\pi}{2}<\|\boldsymbol{\omega}\| \leq 2 \Omega \\ \sqrt{1-\frac{\log \frac{\|\boldsymbol{\omega}\|}{2 \Omega}}{\log \frac{\pi}{2 \Omega}}} & 2 \Omega<\|\boldsymbol{\omega}\| \leq \pi\end{cases}
$$

Their defining property is that they minimize ${ }^{1}$ $\int_{\mathbb{R}^{3}} \boldsymbol{x}^{2}\left|\psi_{\Omega}(\boldsymbol{x})\right|^{2} \mathrm{~d} \boldsymbol{x}$ under the constraint that their bandwidth is limited to $[\Omega, \pi]$ and $\sum_{m \in \mathbb{Z}}\left|\hat{\psi}_{\Omega}\left(2^{m} \boldsymbol{\omega}\right)\right|^{2}=1$.

\subsection{Regional lung texture analysis and UIP classification}

The pulmonary volumes were separated into 36 subregions obtained from all intersections between apical, vertical center, basal, peripheral, left, right, anterior and posterior lung regions [9]. The average energies of the coefficients from each subband $\boldsymbol{E}\left(\mathcal{R}_{i}^{\mathrm{RC}}\left\{\psi_{m} * f\right\}\left(\boldsymbol{x}_{k}\right)\right)$ were computed for each subregion $k$ and concatenated to form the feature vector $\boldsymbol{v}$, where $i=1, \ldots, 3, m=1, \ldots, 4$ and $k=1, \ldots, 36$. The dimension of $\boldsymbol{v}$ is 436, and its construction is illustrated in Fig. 2. The variance of the 3-D Gaussian $g(\boldsymbol{x})$ is set to $1 \mathrm{in} \mathrm{Eq.} \mathrm{(2).}$

A generalized linear model was used to estimate the class membership $\hat{y}$ of $\boldsymbol{v}$ as [17]:

$$
\hat{y}=b_{0}+\boldsymbol{b}^{\boldsymbol{T}} \boldsymbol{v},
$$

where $\operatorname{sign}(\hat{y})>0$ denotes the classic UIP (versus atypical). A 10-fold cross-validation with 200 Monte-Carlo repetitions was used to estimate the generalization performance of our model. The area under the receiver operating characteristic curve (AUC) and classification accuracy (ACC) were computed based on $\hat{y}$ and $\operatorname{sign}(\hat{y})$, respectively.

\footnotetext{
${ }^{1}$ The formula is a very close analytic approximation of the optimal solution that does not have a closed form.
}

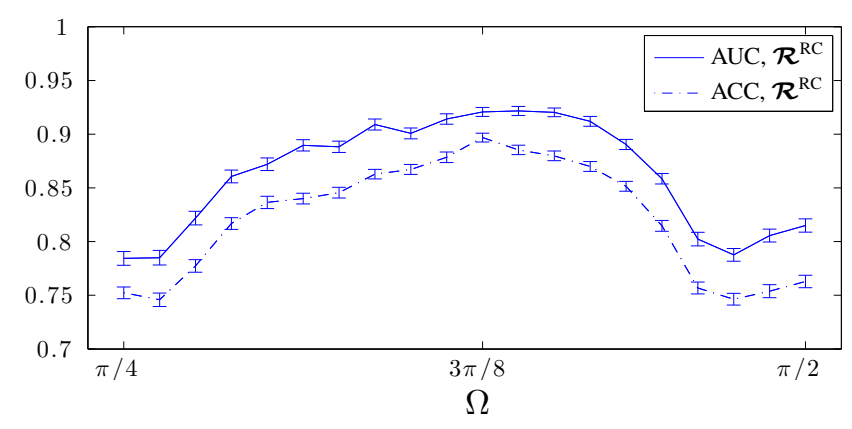

Fig. 3. Classification performance for $\Omega \in[\pi / 4, \pi / 2]$ using the rotation-covariant operator $\mathcal{R}^{\mathrm{RC}}$. The importance of wavelet bandwidth optimization for lung texture analysis is highlighted, where $\Omega=3 \pi / 8$ provided best $\mathrm{AUC}=0.921$ and best ACC $=0.897$.

Table 1. Comparison of $\psi_{\Omega}$ with popular isotropic wavelet pyramid implementations. All are using $\mathcal{R}^{\mathrm{RC}}\left\{\psi_{m} * f\right\}(\boldsymbol{x})$. The AUC reported in our previous study [9] was of 0.811 .

\begin{tabular}{cccc}
\hline \hline Wavelet type & Bandwidth & AUC & ACC \\
\hline Simoncelli [6] & {$[\pi / 4, \pi]$} & $0.775 \pm 0.007$ & $0.741 \pm 0.006$ \\
Meyer [16] & {$[\pi / 4, \pi]$} & $0.834 \pm 0.007$ & $0.8 \pm 0.006$ \\
Papadakis [13] & {$[3 \pi / 10, \pi]$} & $0.901 \pm 0.005$ & $0.86 \pm 0.005$ \\
Proposed $\psi_{\Omega}(3)$ & {$[3 \pi / 8, \pi]$} & $\mathbf{0 . 9 2 1} \pm \mathbf{0 . 0 0 4}$ & $\mathbf{0 . 8 9 7} \pm \mathbf{0 . 0 0 4}$ \\
Shannon [7] & {$[\pi / 2, \pi]$} & $0.802 \pm 0.006$ & $0.758 \pm 0.006$ \\
\hline
\end{tabular}

\section{RESULTS}

The classification performance is reported in Fig. 3 for $\Omega \in$ $[\pi / 4, \pi / 2]$ using the rotation-covariant operator $\mathcal{R}^{\mathrm{RC}}$. The comparison of $\psi_{\Omega}$ with other popular isotropic wavelet pyramids in the literature is detailed in Table 1.

\section{DISCUSSIONS AND CONCLUSIONS}

We proposed an approach for the tuning of radial wavelet bandwidth $[\Omega, \pi], \Omega \in[\pi / 4, \pi / 2]$ of steerable pyramids. The motivation behind this work was to provide a method for finding the minimum spatial support of the wavelet required to leverage the wealth of local texture properties conveyed by modern volumetric medical imaging protocols. The use of the rotation-covariant image operator $\mathcal{R}^{\mathrm{RC}}$ coupled with an optimal wavelet bandwidth of $[3 \pi / 8, \pi]$ provided best performance for the automated classification of UIP with an AUC $=0.921 \pm 0.004$ and an ACC $=0.897 \pm 0.004$. The influence of the radial bandwidth $[\Omega, \pi]$ on the classification performance was found to be large with AUC values in $[0.784,0.921]$ and ACC values in $[0.746,0.897]$ (see Fig. 3), hence demonstrating its importance for leveraging 3D morphological tissue properties in the vicinity of the lung boundaries. The most popular designs for isotropic pyramids (i.e., Simoncelli, Meyer and Shannon) were found to provide 
worst results (see Table 1). This can be explained by their extreme choices of wavelet bandwidth with Shannon being the narrowest (i.e., $[\pi / 2, \pi]$, generating extensive ripple effect in the spatial domain), whereas Simoncelli and Meyer used largest bands (i.e., $[\pi / 4, \pi]$ ). The wavelet proposed by Papadakis (i.e., $[3 \pi / 10, \pi]$ ) yielded near-to-optimal results, which motivated the design of $\psi_{\Omega}$. In fact, the optimal lower bound of the bandwidth of $\psi_{\Omega}$ (i.e., $\Omega=3 \pi / 8$ ) lies exactly in between $\pi / 4$ and $\pi / 2$.

The obtained classification performance suggests that the automated differentiation of classic versus atypical UIP can be done reliably. The performance improvement is large when compared to our previous work: $\mathrm{AUC}=0.811$ in [9] using the same cohort of patients. It can provide valuable computer-aided assistance in less specialized practice centers without access to experts experienced in interstitial lung disease, as well as avoiding unnecessary surgical biopsies. Likewise, we expect the wavelet bandwidth to have a strong influence on 3-D texture classification performance in a wide variety of applications, where little attention has been payed in the literature on its optimization so far.

\section{REFERENCES}

[1] A. Depeursinge, A. Foncubierta-Rodríguez, D. Van De Ville, and H. Müller, "Three-dimensional solid texture analysis and retrieval in biomedical imaging: review and opportunities," Medical Image Analysis, vol. 18, no. 1, pp. 176-196, 2014.

[2] V. A. Kovalev and M. Petrou, "Texture analysis in 3D for tissue characterization," in Handbook of Medical Image Processing and Analysis (Second Edition), I. N. Bankman, Ed., pp. 279-292. Academic Press, Burlington, second edition edition, 2009.

[3] A. Depeursinge, A. Foncubierta-Rodríguez, A. Vargas, D. Van De Ville, A. Platon, P.-A. Poletti, and H. Müller, "Rotation-covariant texture analysis of 4D dual-energy CT as an indicator of local pulmonary perfusion," in IEEE 10th International Symposium on Biomedical Imaging. 2013, ISBI 2013, pp. 149-152.

[4] S. Upadhyay, M. Papadakis, S. Jain, G. Gladish M.D., I. A. Kakadiaris, and R. Azencott, "Semi-automatic discrimination of normal tissue and liver cancer lesions in contrast enhanced X-ray CT-scans," in Abdominal Imaging. Computational and Clinical Applications, pp. 158-167. Springer Berlin Heidelberg, 2012.

[5] J. Fehr and H. Burkhardt, "3D rotation invariant local binary patterns," in 19th International Conference on Pattern Recognition, 2008, ICPR 2008, pp. 1-4.

[6] J. Portilla and E. P. Simoncelli, "A parametric texture model based on joint statistics of complex wavelet coef- ficients," International Journal of Computer Vision, vol. 40, no. 1, pp. 49-70, 2000.

[7] M. Unser, N. Chenouard, and D. Van De Ville, "Steerable pyramids and tight wavelet frames in $L_{2}\left(\mathbb{R}^{d}\right)$," IEEE Transactions on Image Processing, vol. 20, no. 10, pp. 2705-2721, 2011.

[8] G. Raghu, H. R. Collard, J. J. Egan, F. J. Martinez, J. Behr et al., "An official ATS/ERS/JRS/ALAT statement: Idiopathic pulmonary fibrosis: Evidence-based guidelines for diagnosis and management," American Journal of Respiratory and Critical Care Medicine, vol. 183, no. 6, pp. 788-824, 2011.

[9] A. Depeursinge, A. C. Chin, A. N. Leung, D. Terrone, M. Bristow, G. Rosen, and D. L. Rubin, "Automated classification of usual interstitial pneumonia using regional volumetric texture analysis in high-resolution CT," Investigative Radiology, 2015.

[10] P. Pad, V. Uhlmann, and M. Unser, "VOW: Variance optimal wavelets for the steerable pyramid," in IEEE International Conference on Image Processing, ICIP 2014.

[11] N. Chenouard and M. Unser, "3D steerable wavelets and monogenic analysis for bioimaging," in 2011 IEEE International Symposium on Biomedical Imaging: From Nano to Macro, 2011, pp. 2132-2135.

[12] A. Depeursinge, A. Foncubierta-Rodríguez, D. Van De Ville, and H. Müller, "Rotation-covariant texture learning using steerable Riesz wavelets," IEEE Transactions on Image Processing, vol. 23, pp. 898-908, 2014.

[13] J. R. Romero, S. K. Alexander, S. Baid, S. Jain, and M. Papadakis, "The geometry and the analytic properties of isotropic multiresolution analysis," Advances in Computational Mathematics, vol. 31, no. 1-3, pp. 283328, 2009.

[14] S. Held, M. Storath, P. Massopust, and B. Forster, "Steerable wavelet frames based on the riesz transform," IEEE Transactions on Image Processing, vol. 19, no. 3, pp. 653-667, 2010.

[15] M. Papadakis, G. Gogoshin, I. A. Kakadiaris, D. J. Kouri, and D. K. Hoffman, "Nonseparable radial frame multiresolution analysis in multidimensions and isotropic fast wavelet algorithms," in Proc. SPIE Wavelets: Applications in Signal and Image Processing X, 2003, vol. 5207, pp. 631-642.

[16] I. Daubechies, Ten lectures on wavelets, vol. 61, SIAM, 1992.

[17] R. Tibshirani, "Regression shrinkage and selection via the Lasso," Journal of the Royal Statistical Society. Series B (Methodological), vol. 58, pp. 267-288, 1996. 